\title{
Could tear proteins be biomarkers for Alzheimer disease?
}

Given the inaccessibility of the brain, attention has turned to body fluids, such as serum and cerebrospinal fluid, as potential sources of diagnostic and prognostic biomarkers for neurological disease. Now, a study from Hungary has identified a specific combination of proteins in tear fluid that could serve as biomarkers for Alzheimer disease (AD).

"Extensive eye-related complications have been observed in $\mathrm{AD}$, including reduced numbers of retinal ganglion cells, thinning of the nerve cell layer, decline in the number of axons, and amyloid deposition in the lens and retina," explains Éva Csősz, who led the study. "We hypothesized that $\mathrm{AD}$-induced alterations in the eye may provoke alterations in tear production and composition of tear proteins, which could eventually be used as biomarkers for AD diagnosis."

The new study included 14 patients with $\mathrm{AD}$ and nine cognitively healthy controls. The researchers found that tear flow rate was considerably faster in the patients with $\mathrm{AD}$ than in the controls (mean $12 \mu \mathrm{l} / \mathrm{min}$ versus $6 \mu \mathrm{l} / \mathrm{min}$ ). "To the best of our knowledge, this is the first work demonstrating $\mathrm{AD}$-dependent alterations in lacrimal secretion," points out Csősz. In addition, total protein levels were elevated in tears from patients with $\mathrm{AD}$ (mean $8.8 \mu \mathrm{g} / \mu \mathrm{l}$, compared with $4.4 \mu \mathrm{g} / \mu \mathrm{l}$ in controls).

Tear samples from a subset of the study cohort (three patients with AD and two controls) were subjected to analysis by SDS-PAGE and mass spectrometry, so as to identify proteins that were differentially

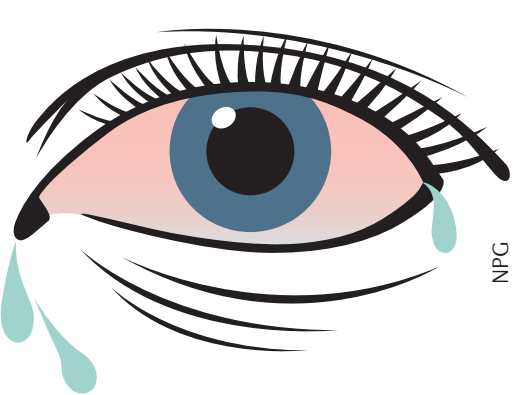

expressed between the two groups. From this analysis, a panel of proteins was earmarked for further investigation in the entire cohort. Using a targeted proteomics approach known as selected reaction monitoring (SRM), the team discovered that a combination of four tear proteins lipocalin-1, dermicidin, lysozyme C and lactritin — had $81 \%$ sensitivity and $77 \%$ specificity for $\mathrm{AD}$.

The researchers now plan to validate these potential biomarkers in larger cohorts, and to establish whether they are specific for $\mathrm{AD}$, or for neurodegeneration in general. "We envisage a bedside test, in which these markers are used as a first screen," says Csősz. "If the test is positive, rigorous examinations can be performed to establish the diagnosis."

\section{Heather Wood}

ORIGINAL ARTICLE Kalló, G. et al. Changes in the chemical barrier composition of tears in Alzheimer's disease reveal potential tear diagnostic biomarkers. PLoS ONE 11, e0158000 (2016) 